\title{
BMJ Open Exercise Programme in Endometrial Cancer; Protocol of the Feasibility and Acceptability Survivorship Trial (EPEC-FAST)
}

\author{
Anke Smits, ${ }^{1}$ Alberto Lopes, ${ }^{1}$ Nagindra Das, ${ }^{1}$ Ruud Bekkers, ${ }^{2}$ Leon Massuger, ${ }^{2}$ \\ Khadra Galaal ${ }^{1}$
}

To cite: Smits A, Lopes A Das N, et al. Exercise Programme in Endometrial Cancer; Protocol of the Feasibility and Acceptability Survivorship Trial (EPEC-FAST). BMJ Open 2015;5:e009291. doi:10.1136/bmjopen-2015009291

- Prepublication history for this paper is available online. To view these files please visit the journal online (http://dx.doi.org/10.1136/ bmjopen-2015-009291).

Received 2 July 2015 Revised 23 September 2015 Accepted 24 September 2015

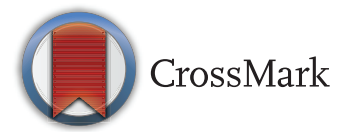

${ }^{1}$ Department of Gynaecology, Royal Cornwall Hospital Trust, Truro, Cornwall, UK ${ }^{2}$ Department of Gynaecology, Radboud UMC, Nijmegen, The Netherlands

Correspondence to Khadra Galaal;

khadra.galaal@rcht.cornwall. nhs.uk, khadragalaal@yahoo. co.uk

\section{ABSTRACT}

Introduction: Obesity has been associated with impaired quality of life and poorer outcomes in endometrial cancer survivors. Lifestyle interventions promoting exercise and weight reduction have been proposed for survivorship care. However, studies evaluating exercise programmes for endometrial cancer survivors are lacking.

Purpose: The objective of this study is to evaluate the feasibility of an individualised exercise intervention for endometrial cancer survivors to improve quality of life. Methods and analysis: This is a feasibility study in which women will undergo a 10-week exercise programme with a personal trainer. The study population comprises women with confirmed diagnosis of endometrial cancer, who have completed surgical treatment with curative intent, and are aged 18 years or older. The study will take place at the Royal Cornwall Hospital Trust, UK. Feasibility will be evaluated in terms of recruitment, adherence and compliance to the programme. Secondary outcomes are quality of life, psychological distress, fatigue, pain and complication rates. In addition, the acceptability of the programme will be assessed.

Ethics and dissemination: Ethical approval was obtained through the Exeter NRES Committee. The study results will be used to optimise the intervention content, and may serve as the foundation for a larger definitive trial. Results will be disseminated through peer-review journals, congresses, relevant clinical groups and presented on the Trust's website.

Trial registration number: NCT02367950; pre-results.

\section{BACKGROUND}

Endometrial cancer is the most common form of gynaecological cancer in the UK, with 8475 new cases being diagnosed in 2011 alone. ${ }^{1}$ Endometrial cancer has a relatively good prognosis, with a 10 -year survival rate of $78 \%$, resulting in a large group of longterm survivors. ${ }^{1}$ Subsequently, health-related quality of life is now recognised as an important outcome for endometrial cancer survivors.

The majority of women diagnosed with endometrial cancer are obese, as excess weight is an important risk factor for endometrial cancer. ${ }^{2}{ }^{3}$ In addition, few endometrial cancer survivors meet the health recommendations for physical activity. ${ }^{4}$ Obesity and inactivity have been identified as significant factors negatively influencing the quality of life of endometrial cancer survivors, surpassing the negative influence of the physical and psychological stress that comes with cancer diagnosis and treatment alone. $^{5}{ }^{6}$ In addition, obese endometrial cancer survivors are at risk of numerous obesity-related comorbidities and possibly poorer survival. ${ }^{7} 8$

It has been suggested that exercise and weight reduction may be viable means to improve the quality of life and other health-related outcomes of cancer survivors. ${ }^{9}{ }^{10}$ In addition, the American College of Sports Medicine (ACSM) has recommended exercise training for patients with cancer, stating that it is safe and benefits functioning and quality of life. ${ }^{11}$ However, previous research has shown that endometrial cancer survivors struggle to achieve this on their own as they do not engage in a healthy lifestyle and find it difficult to implement lifestyle changes. ${ }^{412}$

The feasibility of some lifestyle interventions, including multidisciplinary counselling, behavioural change interventions and a home-based exercise programme, has been demonstrated in endometrial cancer survivors. ${ }^{13-16}$ Thus far, relatively few studies have been performed to test the potential usefulness of an exercise intervention to improve the quality of life and other health outcomes of endometrial cancer survivors. In addition, 
the essential components and attributes of an intervention, including mode, intensity, frequency and duration of an exercise programme, have not yet been established..$^{10}$ To the best of our knowledge, an individualised exercise intervention has not yet been evaluated.

We, therefore, want to evaluate the feasibility of introducing an individualised exercise programme in the care for endometrial cancer survivors to improve quality of life and other health outcomes.

\section{METHODS}

Design

This is a single-arm prospective feasibility study to evaluate the introduction of an individualised exercise intervention for endometrial cancer survivors. In addition, a qualitative evaluation will be performed to assess the acceptability of the intervention. The study will take place at the Royal Cornwall Hospital Trust.

\section{Study population}

The eligibility criteria are: women older than 18 years, with a diagnosis of primary endometrial cancer, undergoing surgical treatment with curative intent. Exclusion criteria are: women presenting with recurrent endometrial cancer, those with a concurrent cancer, patients unable to give informed consent, those women undergoing palliative treatment. Women with recurrent cancer or receiving treatment in the palliative setting will not be asked to participate because of the burden of the study, and as surgical treatment is usually not a standard component of palliative patient care in endometrial cancer.

\section{Recruitment}

A member of the care team will identify potentially eligible women after confirmation of diagnosis and multidisciplinary team (MDT) review (figure 1). Study posters will be displayed in the gynaecological outpatient clinic and an information leaflet will be provided during admission for surgery. Women will be contacted 1 week after they have been discharged to discuss the study in detail. Women who express an interest will be seen during their postoperative check-up and consented for the study. Women will be recruited over a 12-month period; we aim to recruit a minimum of 15 patients.

\section{Intervention}

The exercise programme consists of $60 \mathrm{~min}$ individualised (one-to-one) training sessions with a personal trainer, once a week, for 10 consecutive weeks (table 1 ). The training sessions will be delivered by one personal trainer to provide consistency. The programme begins after the patient has had her 6-week postoperative check-up, and will take place at a local gym facility. The programme will be tailored to the individual patient through a pre-exercise health assessment, taking into

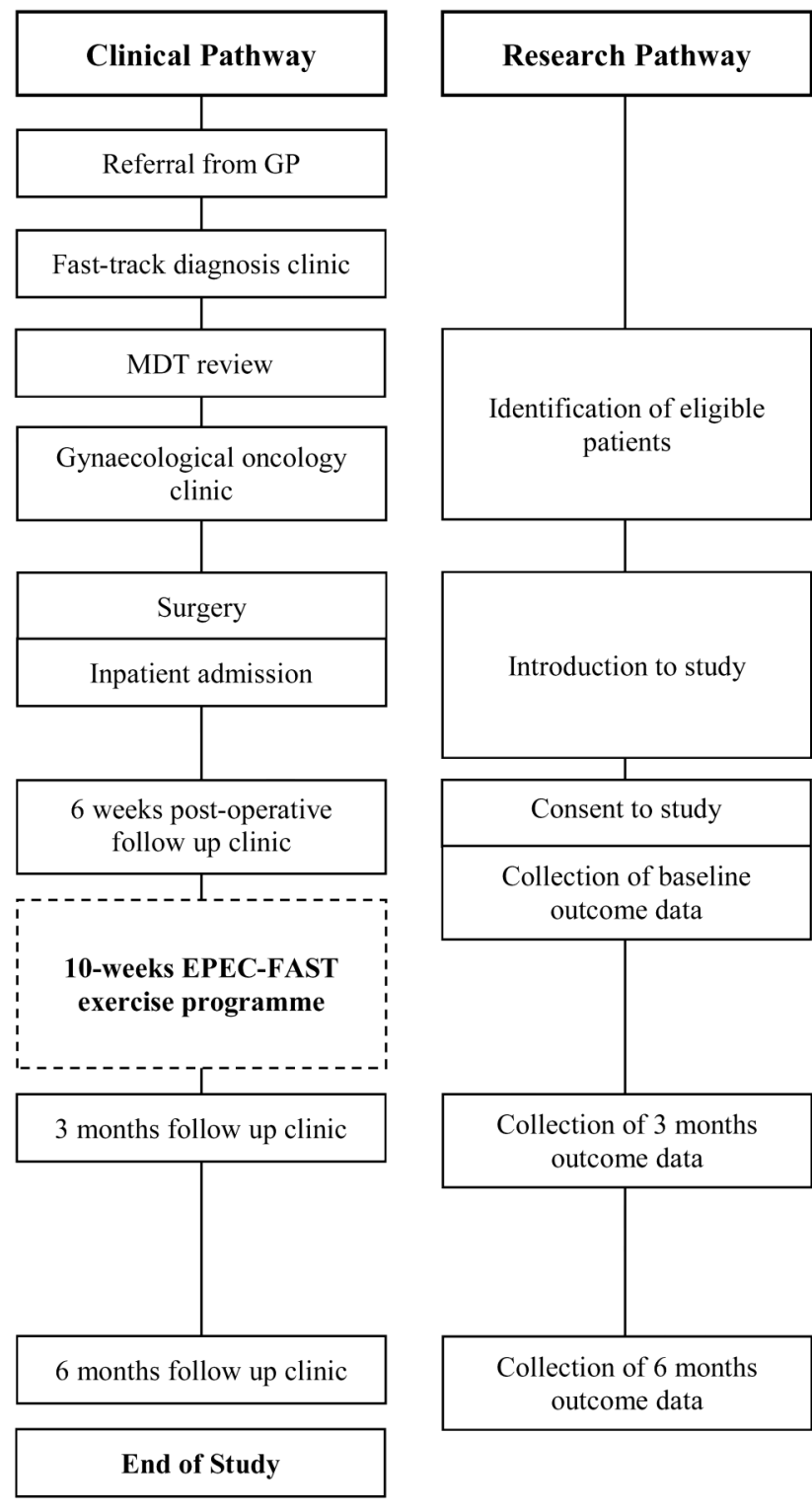

Figure 1 Flow diagram of clinical and research pathway of study. GP, general practitioner; MDT, multidisciplinary team.

consideration their current health status, physical activity level, comorbidities and medical history. Each session consists of a 10 min warm-up, a 40 min workout, consisting of aerobic (cardiovascular) exercise, pillar strength exercise (including hip and core stability), and resistance training (muscular strength and endurance training), and a $10 \mathrm{~min}$ cool down, including flexibility training. The exercise phase will be performed at a level of 40-60\% maximum heart rate (MHR), measured with a Polar Heart Rate monitor using the Karvonen method for calculating the target heart rate interval. The resistance training will be performed at an intensity of 40 $60 \%$ with one repetition maximum (1 RM). In the case of potential adverse effects, such as a strain, injury or exacerbation of other symptoms, the session will be discontinued and the participant will be referred for medical attention, if needed. Participants will receive 
Table 1 The EPEC-FAST Exercise programme

\begin{tabular}{|c|c|}
\hline $\begin{array}{l}\text { Exerci } \\
\text { phase }\end{array}$ & Details \\
\hline $\begin{array}{l}\text { Warm-up } \\
10 \text { min }\end{array}$ & $\begin{array}{l}\text { Low intensity warm-up using an exercise } \\
\text { bike or a treadmill }\end{array}$ \\
\hline $\begin{array}{l}\text { Exercise } \\
40 \mathrm{~min}\end{array}$ & 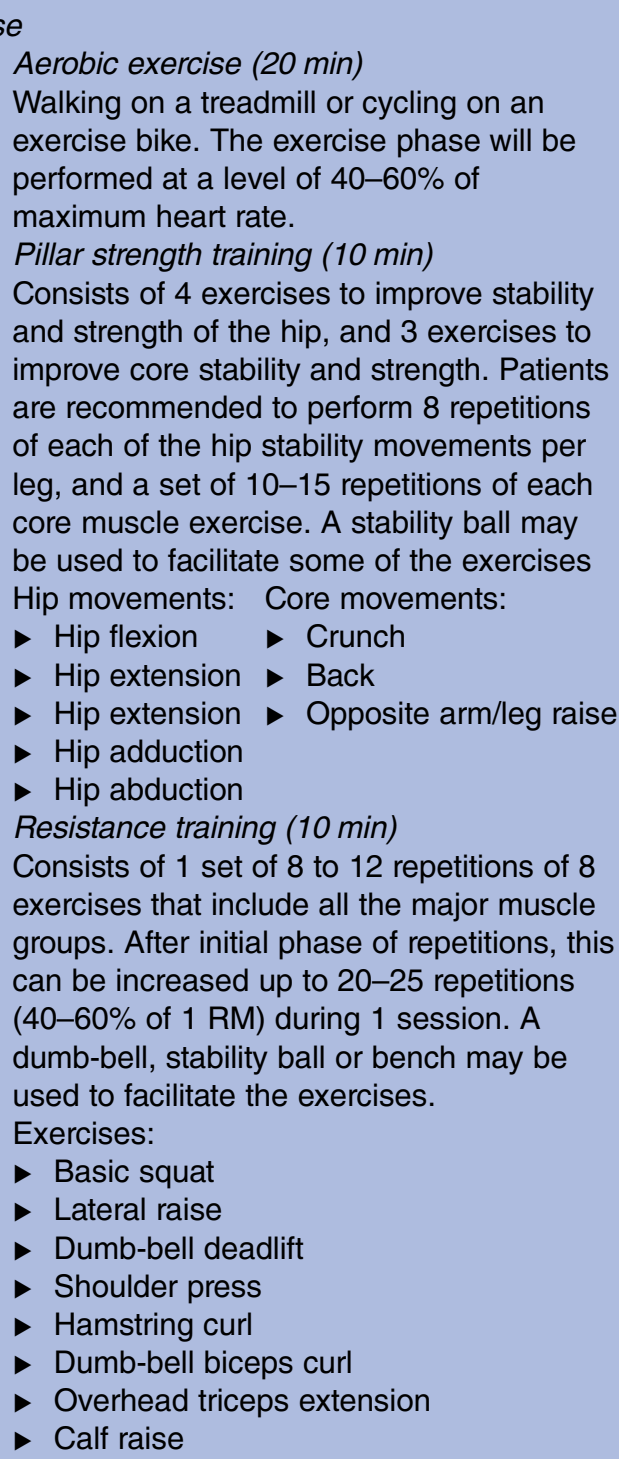 \\
\hline
\end{tabular}

Cool down

$10 \mathrm{~min} \quad$ Set of 6 stretching and flexibility exercises. Four repetitions of each of the following muscle groups will be performed for10-

$30 \mathrm{~s}$.

- Lower back

- Tensor fasciae latae

- Hip flexor

- Quadriceps

- Hamstring

- Calf

*The content of the programme will subject to individual variability and will be adjusted to the individual patient.

general physical activity recommendations for moderate-intensity exercise for $150 \mathrm{~min}$ per week as part of standard practice. ${ }^{11} 17$

\section{Outcomes}

Outcomes are assessed at baseline at the completion of the intervention, and 3 months after completion of the intervention. Figure 1 demonstrates the pathways of the study, including data collection.

\section{Primary outcome}

The primary outcome of this study will be its feasibility aspects, including willingness of clinicians to recruit patients, number of eligible patients and recruitment rates. These will be obtained from hospital medical files as the clinical care team will document eligibility and recruitment. Willingness of clinicians to recruit will be assessed through interviews with the clinical care team. Adherence and compliance rates to the programme will be collected from registration forms used during the exercise programme. Reasons for non-adherence or non-compliance will be identified, and further addressed in the qualitative evaluation.

\section{Secondary outcomes}

Several measures evaluating possible outcomes for a definitive trial will be assessed at baseline, postintervention (3 months) and 3 months after completion (at 6 months) to provide characteristics and SD. Quality of life is evaluated through the internationally established EORTC QLQ-C30 and the QLQ-EN24 questionnaires. ${ }^{18}{ }^{19}$ The QLQ-C30 is a cancer-specific questionnaire covering several areas of quality of life: physical, role, emotional, cognitive and social functioning, as well as symptom distress and global quality of life. ${ }^{18}$ The QLQ-EN24 is a specific module for endometrial patients with cancer, covering specifically relevant issues such as body image, sexuality and treatment symptoms. $^{19}$ The Hospital Anxiety and Depression Scale (HADS) is used to assess psychological distress, and fatigue and pain are measured through the Brief Fatigue Inventory (BFI) and the Brief Pain Inventory (BPI). ${ }^{20-22}$ Permissions are obtained for all measures used during the study.

Participants will receive a physical fitness assessment at baseline and at 3 months, which includes their current health and measured weight and height, body mass index (BMI), waist circumference, body fat percentage, lean muscle tissue, resting metabolism and the 6 min walk test. The $6 \mathrm{~min}$ walk test will be performed on a treadmill, using an adaptation of the American Thoracic guidelines. $^{23}$ Weight and BMI will also be collected at 6 months. Other outcomes, such as adverse outcomes, will be collected throughout the trial.

\section{Qualitative evaluation}

A qualitative evaluation will be undertaken after completion of the exercise programme. This will be through one-on-one telephone interviews using a moderator guide for a selected subgroup of the study population. Open-ended questions will be used to encourage reflection and elaboration on different aspects of the 
exercise programme. The interviews will be recorded and transcribed, and data analysis of the interviews will occur through content analysis using simple descriptive thematic analyses, which will be performed by a researcher independent of the research delivery team. ${ }^{24}$ All data will be prospectively collected in an electronic database.

\section{Data collection}

The quantitative measures will be collected by a member of the clinical care team at three time points: baseline, after completion of the exercise programme (3 months) and 3 months after completion (at 6 months). Questionnaires will be completed during standard clinical visits, which follow the same time points. The qualitative element of the study will be undertaken after completion of the exercise programme. We aim to evaluate approximately 8-10 participants through individual semistructured interviews. This will be a purposively selected population with maximum variation in terms of age, BMI, adherence and adverse events.

\section{Statistical analysis}

Data will be presented detailing the number of patients who were approached, the number who were eligible, and the number providing consent. As this is a feasibility study, no power calculation has been performed. Compliance rates at all stages will be presented: the number of exercise sessions undertaken (mean, median and full distribution), the number of questionnaires completed at each stage and more generally, the completeness of data on all outcomes at each time point. Participating patients' characteristics (demographics, comorbidities, clinical details) will be summarised and where possible, compared with the overall population of relevant patients to explore possible factors associated with participation. Where possible, the reasons will be ascertained for potentially eligible patients not being approached to consider participation.

The questionnaire outcomes (EORTC QLQ-C30 +QLQ-EN24, HADS, BPI and BFI) will be analysed according to scoring procedures and will be linearly transformed into scales. The analysis for the outcome of quality of life, measured with the EORTC QLQ-C30 and QLQ-EN24 questionnaire is based on standard scoring procedures. $^{25}$ Data will be presented on means (or medians, as appropriate) and SDs at each time point, plus correlations and changes between baseline and follow-up scores (to inform future sample size calculations for a potential randomised trial).

\section{Ethics and dissemination}

The study results will be used to optimise the intervention content and may serve as the foundation for a larger definitive trial. We aim to disseminate the results through peer-review journals, presentation at international conferences, relevant clinical groups and results will be presented on the Trust's website.

\section{DISCUSSION}

The aim of this article was to describe the protocol of a feasibility study evaluating an individualised exercise intervention in the management of endometrial cancer survivors to improve their quality of life and other health-related outcomes.

Research has shown that the majority of endometrial cancer survivors do not meet recommendations for physical activity, have poorer fitness, and are usually overweight or obese..$^{242627}$ BMI and physical activity levels have been extensively linked to the quality of life of endometrial patients with cancer; thus, BMI and physical activity are independent factors impacting quality of life. ${ }^{5} 62728$ Furthermore, it has been hypothesised that physical activity may protect from the negative impact of a higher BMI on quality of life outcomes. ${ }^{27}$

The intervention consists of 10 individualised (one-to-one) training sessions with a personal trainer at a local gym. The content of the intervention was based on national and cancer-specific recommendations of the American College of Sports Medicine, evidence from the literature, and feedback from relevant patient groups. ${ }^{11} 172930$ The ACSM has concluded that exercise during and after cancer treatment is safe and should be encouraged, although prescriptions should be individualised according to the patient. Therefore, the EPEC-FAST programme will be tailored to the individual patient taking into consideration their current health status, physical activity level, comorbidities and medical history though a pre-exercise medical assessment following the ACSM recommendations. ${ }^{11}$

Unfortunately, the ACSM was unable to make recommendations for exercise interventions in gynaecological patients with cancer specifically due to limited data. Therefore, general recommendations for patients with cancer were followed when developing the EPEC-FAST programme to include aerobic fitness, strength exercises (pillar strength and resistance training), and flexibility training. ${ }^{11}$ The frequency (once a week) and duration ( 10 weeks) of the programme were largely based on input from patient groups, as we could not find concrete guidance on essential components and attributes of an exercise programme for endometrial patients with cancer in the literature. ${ }^{10} 11$

Important strengths of the intervention include the individualised programme and the one-to-one sessions in a private gym; these have not as yet been evaluated in endometrial patients with cancer. Attainable goals and individual guidance are known to improve adherence and compliance to a programme. ${ }^{30}$ We believe this is specifically applicable for endometrial patients with cancer with high BMI, as they experience poorer body image and self-esteem after diagnosis and treatment. ${ }^{31}{ }^{32}$ Third, we consider the timing of the intervention to be 
advantageous as women are in a teachable moment, and are interested in modifying their lifestyle in the hope of achieving improved health. ${ }^{33}{ }^{34}$ In addition, the intervention fits within the current care pathway, being less burdensome for patients as they do not have to undertake additional clinical visits.

A limitation of the protocol is that the effect of the programme on overall physical activity behaviour is not assessed. We recommend this to be included as an outcome in a definitive trial, possibly through selfreported measures or objective measures such as accelerometers.

\section{CONCLUSION}

In this article, we described the protocol of an intervention aimed at improving physical fitness, quality of life and other health outcomes in endometrial cancer survivors. In addition, we presented the study design to investigate its feasibility of delivering the intervention within the current healthcare model. The results of the feasibility study may be used for optimisation of the intervention and may serve as a basis for implementation of the intervention in a randomised controlled trial.

Acknowledgements The authors are very grateful to Steve Winnan for designing and delivering the exercise programme, and to Jeannette Preston, Founder of Pants Cancer Charity, for her dedication and support for women with gynaecological cancer. This study was supported by the research and development department of the Royal Cornwall Hospital Trust. The authors are very grateful to Joanne Palmer, Colin Pritchard and Andy Barton for their help with the design of the study. The authors are thankful to Chris Cannaby and Nick Morley for their support. In addition, the authors would like to thank the following members of the Royal Cornwall Hospital Trust clinical team: Emma Arthur, Emma Kent, Hayley Carey, Sami Bishieri, Sonia Thomas, Julie Hill and Alison Callaway.

Contributors AS, AL and KG were responsible for conception and design of the study protocol, drafting and revising of the article. ND undertook conception and design of the study protocol and revising of the article. RB was responsible for design of the study protocol and revising of the article. LM was responsible for revising of the article.

Funding This work was supported by Pants Cancer Charity.

Competing interests None declared.

Ethics approval Exeter NRES Committee.

Provenance and peer review Not commissioned; externally peer reviewed.

Open Access This is an Open Access article distributed in accordance with the Creative Commons Attribution Non Commercial (CC BY-NC 4.0) license, which permits others to distribute, remix, adapt, build upon this work noncommercially, and license their derivative works on different terms, provided the original work is properly cited and the use is non-commercial. See: http:// creativecommons.org/licenses/by-nc/4.0/

\section{REFERENCES}

1. Cancer Research UK. Uterine cancer key facts 2011 [updated 07-05-014; cited 2015 June].

2. Smits A, Lopes A, Das N, et al. The impact of BMI on quality of life in obese endometrial cancer survivors: does size matter? Gynecol Oncol 2014;132:137-41.

3. Jenabi E, Poorolajal J. The effect of body mass index on endometrial cancer: a meta-analysis. Public Health 2015;129: 872-80.
4. von Gruenigen VE, Waggoner SE, Frasure HE, et al. Lifestyle challenges in endometrial cancer survivorship. Obstet Gynecol 2011;117:93-100.

5. Smits A, Lopes A, Bekkers R, et al. Body mass index and the quality of life of endometrial cancer survivors-a systematic review and meta-analysis. Gynecol Oncol 2015;137:180-7.

6. Koutoukidis DA, Knobf MT, Lanceley A. Obesity, diet, physical activity, and health-related quality of life in endometrial cancer survivors. Nutr Rev 2015;73:399-408.

7. Arem $\mathrm{H}$, Irwin ML. Obesity and endometrial cancer survival: a systematic review. Int J Obes (Lond) 2013;37:634-9.

8. [No authors listed]. Clinical guidelines on the identification, evaluation, and treatment of overweight and obesity in adults-the evidence report. National Institutes of Health. Obes Res 1998;6 (Suppl 2):51S-209S.

9. Kolotkin RL, Crosby RD, Williams GR, et al. The relationship between health-related quality of life and weight loss. Obes Res 2001;9:564-71.

10. Mishra SI, Scherer RW, Geigle PM, et al. Exercise interventions on health-related quality of life for cancer survivors. Cochrane Database Syst Rev 2012;8:CD007566.

11. Schmitz KH, Courneya KS, Matthews $C$, et al. American College of Sports Medicine roundtable on exercise guidelines for cancer survivors. Med Sci Sports Exerc 2010;42:1409-26.

12. Beesley VL, Eakin EG, Janda M, et al. Gynecological cancer survivors' health behaviors and their associations with quality of life. Cancer Causes Control 2008;19:775-82.

13. von Gruenigen VE, Courneya KS, Gibbons HE, et al. Feasibility and effectiveness of a lifestyle intervention program in obese endometria cancer patients: a randomized trial. Gynecol Oncol 2008;109:19-26.

14. von Gruenigen V, Frasure $\mathrm{H}$, Kavanagh MB, et al. Survivors of uterine cancer empowered by exercise and healthy diet (SUCCEED): a randomized controlled trial. Gynecol Oncol 2012;125:699-704.

15. Donnelly CM, Blaney JM, Lowe-Strong A, et al. A randomised controlled trial testing the feasibility and efficacy of a physical activity behavioural change intervention in managing fatigue with gynaecological cancer survivors. Gynecol Oncol 2011;122:618-24.

16. Basen-Engquist $\mathrm{K}$, Carmack $\mathrm{C}$, Brown $\mathrm{J}$, et al. Response to an exercise intervention after endometrial cancer: differences between obese and non-obese survivors. Gynecol Oncol 2014:133:48-55.

17. Bull FC. and the Expert Working Groups. Physical Activity Guidelines in the U.K.: Review and Recommendations. School of Sport, Exercise and Health Sciences, Loughborough University, May 2010.

18. Aaronson NK, Ahmedzai S, Bergman B, et al. The European Organization for Research and Treatment of Cancer QLQ-C30: a quality-of-life instrument for use in international clinical trials in oncology. J Natl Cancer Inst 1993;85:365-76.

19. Greimel E, Nordin A, Lanceley A, et al. Psychometric validation of the European Organisation for Research and Treatment of Cancer Quality of Life Questionnaire-Endometrial Cancer Module (EORTC QLQ-EN24). Eur J Cancer 2011;47:183-90.

20. Zigmond AS, Snaith RP. The hospital anxiety and depression scale. Acta Psychiatr Scand 1983;67:361-70.

21. Mendoza TR, Wang XS, Cleeland CS, et al. The rapid assessment of fatigue severity in cancer patients: use of the Brief Fatigue Inventory. Cancer 1999;85:1186-96.

22. Cleeland CS, Ryan KM. Pain assessment: global use of the Brief Pain Inventory. Ann Acad Med Singapore 1994;23:129-38.

23. ATS Committee on Proficiency Standards for Clinical Pulmonary Function Laboratories. ATS statement: guidelines for the six-minute walk test. Am J Respir Crit Care Med. 2002;166:111-17.

24. Braun V, Clarke V. Using thematic analysis in psychology. Qualitative Research in Psychology, 3, 2. 2006:77-101.

25. Fayers PM AN, Bjordal K, Groenvold M, et al. on, Group. botEQoL. The EORTC QLQ-C30 scoring manual. 3rd edn. Brussels: European Organisation for Research and Treatment of Cancer, 2001.

26. Peel $A B$, Barlow $C E$, Leonard $D$, et al. Cardiorespiratory fitness in survivors of cervical, endometrial, and ovarian cancers: the cooper center longitudinal study. Gynecol Oncol 2015;138:394-7.

27. Lin LL, Brown JC, Segal S, et al. Quality of life, body mass index, and physical activity among uterine cancer patients. Int $J$ Gynecol Cancer 2014;24:1027-32.

28. Courneya KS, Karvinen $\mathrm{KH}$, Campbell KL, et al. Associations among exercise, body weight, and quality of life in a population-based sample of endometrial cancer survivors. Gynecol Oncol 2005;97:422-30.

29. Bull FC and the Expert Working Group. Physical Activity Guidelines in the U.K.: Review and Recommendations. School of Sport, 
Exercise and Health Sciences, Loughborough University, Appendices. 2010.

30. Bourke L, Homer KE, Thaha MA, et al. Interventions for promoting habitual exercise in people living with and beyond cancer. Cochrane Database Syst Rev 2013;9:CD010192.

31. Juraskova I, Butow P, Robertson R, et al. Post-treatment sexual adjustment following cervical and endometrial cancer: a qualitative insight. Psychooncology 2003;12:267-79.

32. Kullmer U, Stenger K, Milch W, et al. Self-concept, body image, and use of unconventional therapies in patients with gynaecological malignancies in the state of complete remission and recurrence. Eur J Obstet Gynecol Reprod Biol 1999;82:101-6.

33. Demark-Wahnefried W, Aziz NM, Rowland JH, et al. Riding the crest of the teachable moment: promoting long-term health after the diagnosis of cancer. J Clin Oncol 2005;23: 5814-30.

34. Stull VB, Snyder DC, Demark-Wahnefried W. Lifestyle interventions in cancer survivors: designing programs that meet the needs of this vulnerable and growing population. J Nutr 2007;137(1 Suppl): 243S-8S. 